\title{
BMJ Open Primary Sjogren's syndrome and the risk of acute pancreatitis: a nationwide cohort study
}

\author{
Chi-Ching Chang, ${ }^{1,2}$ Yu-Sheng Chang, ${ }^{2,3}$ Shu-Hung Wang, ${ }^{3}$ Shyr-Yi Lin, ${ }^{4,5}$ \\ Yi-Hsuan Chen, ${ }^{6}$ Jin Hua Chen ${ }^{6,7}$
}

To cite: Chang $\mathrm{C}-\mathrm{C}$, Chang Y-S, Wang S-H, et al. Primary Sjogren's syndrome and the risk of acute pancreatitis: a nationwide cohort study. BMJ Open 2017;7:e014807. doi:10.1136/ bmjopen-2016-014807

- Prepublication history is available. To view these files please visit the journal online (http://dx.doi.org/10.1136/ bmjopen-2016-014807).

Received 19 0ctober 2016 Revised 11 April 2017 Accepted 11 April 2017

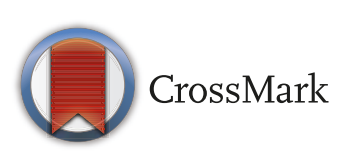

For numbered affiliations see end of article.

Correspondence to Professor Jin Hua Chen; jh_chen@tmu.edu.tw

\section{ABSTRACT}

Objective Studies on the risk of acute pancreatitis in patients with primary Sjogren's syndrome (pSS) are limited. We evaluated the effects of pSS on the risk of acute pancreatitis in a nationwide, population-based cohort in Taiwan.

Study design Population-based retrospective cohort study.

Setting We studied the claims data of the $>97 \%$ Taiwan population from 2002 to 2012.

Participants We identified 9468 patients with pSS by using the catastrophic illness registry of the National Health Insurance Database in Taiwan. We also selected 37872 controls that were randomly frequency matched by age (in 5 year bands), sex and index year from the general population.

Primary outcome measure We analysed the risk of acute pancreatitis by using Cox proportional hazards regression models including sex, age and comorbidities.

Results From 23.74 million people in the cohort, 9468 patients with pSS ( $87 \%$ women, mean age $=55.6$ years) and 37872 controls were followed-up for 4.64 and 4.74 years, respectively. A total of 44 cases of acute pancreatitis were identified in the pSS cohort versus 105 cases in the non-pSS cohort. Multivariate Cox regression analysis indicated that the incidence rate of acute pancreatitis was significantly higher in the pSS cohort than in the non-pSS cohort (adjusted HR (aHR) 1.48, 95\% Cl 1.03 to 2.12). Cyclophosphamide use increased the risk of acute pancreatitis (aHR $5.27,95 \% \mathrm{Cl} 1.16$ to 23.86). By contrast, hydroxychloroquine reduced the risk of acute pancreatitis (aHR $0.23,95 \% \mathrm{Cl} 0.09$ to 0.55 ).

Conclusion This nationwide, retrospective cohort study demonstrated that the risk of acute pancreatitis was significantly higher in patients with pSS than in the general population.

\section{INTRODUCTION}

Sjogren's syndrome (SS) is a slowly progressive systemic autoimmune disease that may present either alone as primary SS (pSS) or, in association with an underlying autoimmune disease, as secondary SS. Systemic manifestations may result from cutaneous, respiratory, renal, hepatic, neurologic and vascular involvement. ${ }^{1}$ However, it mainly

\section{Strengths and limitations of this study}

- This is the first nationwide population-based cohort study that demonstrated the patients with Sjogren's syndrome increase the risk of acute pancreatitis.

- Data on alcoholism and smoking, the major risk factors of acute pancreatitis, were unavailable in our study. However, no reports have mentioned the relationship between primary Sjogren's syndrome (pSS) and alcoholism.

- Because of the lack of data on the severity of pSS, laboratory results and indications for medication use, we could not determine the mechanism of pancreatitis.

affects the salivary and lachrymal glands and leads to keratoconjunctivitis sicca and xerostomia because of focal inflammation. ${ }^{2}$ The pancreas is, in part, an exocrine gland that is functionally and histologically comparable to the salivary glands. Involvement of pancreatic dysfunction in SS has been hypothesised. ${ }^{3}$

Acute pancreatitis, an inflammatory disorder of the pancreas, is the leading cause of admission for gastrointestinal disorders and may be fatal or lead to severe complications in certain cases. In addition to typical risk factors such as ageing, alcoholism, smoking, gallstone, anatomic abnormalities and metabolic factors, patients with autoimmune diseases have been shown to have a higher risk of autoimmune pancreatitis (AIP). ${ }^{4}$ Many reports have mentioned the association between pSS and $\mathrm{AIP}^{5-7}$ In the largest series of patients with pSS (1010 patients), the prevalence of acute pancreatitis was $0.5 \%{ }^{8}$ Despite these case reports and the case series of pSS-related acute pancreatitis, ${ }^{89}$ no cohort study has evaluated the risk of acute pancreatitis in patients with pSS. This risk should be assessed in a large population because of the low incidence rate.

Taiwan's National Health Insurance (NHI), a mandatory universal health insurance 
programme, was began in 1995 and offers comprehensive medical care coverage almost for all Taiwanese residents. The validity of the NHI Database in Taiwan has been evaluated, and research articles have been accepted worldwide for public access. ${ }^{10-12}$ By using the NHI dataset, we conducted a nationwide cohort study to investigate the risk of acute pancreatitis in patients with pSS and related risk factors.

\section{METHODS}

\section{Data source}

The cohort from NHI database was analysed in this study. The bureau of NHI in Taiwan maintains a research-oriented database through the Health and Welfare Statistics Application Center (HWSAC) of the Ministry of Health and Welfare; this database includes all the original claims healthcare data of $>97 \%$ of the entire Taiwanese population (23.74 million people). Comprehensive information on insurants, including demographic data, dates of clinical visits and disease diagnoses, is included in the database. The diagnostic codes used were based on the International Classification of Diseases, Ninth Revision, Clinical Modification (ICD-9-CM). We studied the data of the Taiwanese population from 2002 to 2012. The study was approved by the Institutional Review Board of Taipei Medical University (approval number: N201509007). As the datasets used in this study consist of de-identified secondary data released to the public for research purpose, no consent was needed for the review by the ethical review board.

\section{Study population and design}

In Taiwan, rheumatologists can apply for a catastrophic illness card for any patient with SS who fulfils the criteria of the American-European Consensus Group (AECG) for SS. ${ }^{3}$ The application of the catastrophic illness card is scrutinised in a peer review process. SS patients with the catastrophic illness card can be exempted from copayment. We used the Registry for Catastrophic Illness Patients in NHI database to identify SS (ICD-9-CM Code 710.2) patients in the claims data, and the first-time SS diagnosis served as the index date from 2002 to 2012. In addition, we excluded patients with comorbidities such as systemic lupus erythematous, rheumatoid arthritis, scleroderma, polymyositis and dermatomyositis to limit our study sample to pSS. Patients with pSS and comparison controls (non-pSS) were frequency matched at a 1:4 ratio by age (in 5-year bands), sex and index year.

\section{Outcome measures and case identification}

The primary outcome was newly diagnosed acute pancreatitis from hospitalisation records. All participants were followed-up from the index date to the date of the primary outcome, withdrawal from the NHI programme, or the end of 2012, whichever came first. We identified patients with a discharge diagnosis of acute pancreatitis (ICD-9-CM Code 577.0 in any position of the five diagnosed codes). To overcome this misclassification bias, we included only patients who had been hospitalised to minimise false positive cases. In studies using the same database, the positive predictive value was high $(90 \%)$ among randomly selected hospitalised patients coded with acute pancreatitis. ${ }^{11} 12$

Patients who had been diagnosed with acute pancreatitis before the index date or chronic pancreatitis (ICD-9-CM Code 577.1) and those with incomplete age or sex information were excluded from this study. In Taiwan, the medical reimbursements and discharge notes of acute pancreatitis patients are scrutinised in a peer review process.

\section{Exposure variables}

In addition to pSS, demographic characteristics such as sex, age and comorbidities were analysed. Pre-existing comorbidities included diabetes mellitus (ICD-9-CM Code 220), hyperlipidaemia (ICD-9-CM Codes 272.0272.4), hypertriglyceridaemia (ICD-9-CM Code 272.1), alcoholism (ICD-9-CM Codes 291, 303, 305-305.03, 571.0571.3), gallstones (ICD-9-CM Codes 574.10 or 574.20), hepatitis B (ICD-9-CM Codes 070.2 or 070.3) and hepatitis C (ICD-9-CM Codes 070.4 or 070.5). Furthermore, we examined the potential effects of common therapies for pSS, including disease-modifying antirheumatic drugs (DMARDs; hydroxychloroquine, sulfasalazine, methotrexate, cyclophosphamide, ciclosporin, mycophenolate mofetil and azathioprine) and steroids. Each medication was assessed as a time-dependent covariate constructed according to the prescription for each month. The drug exposure status was set to 0 if no prescription was filled during the period and set to 1 if at least one prescription was filled during the period. Steroids were analysed as the average daily dose equivalent to prednisolone during the study period.

\section{Statistical analysis}

The SAS 9.3 statistical package was used to perform all analyses in this study. We examined differences in continuous variables between the two cohorts by using a Student's t-test and we examined differences in dichotomous variables of the potential confounders between the two cohorts by using a Pearson $\chi^{2}$ test.

The incidence rate is expressed per 100000 personyears. The cumulative incidence of acute pancreatitis was assessed using the Kaplan-Meier estimator, with significance based on the log-rank test. The Cox proportional hazard regression model was used to analyse the risk of acute pancreatitis. Age, sex and baseline comorbidities were adjusted in multivariate analysis. Crude and adjusted HRs are presented along with $95 \%$ CIs. Each type of drug was separately analysed as a time-dependent effect in the Cox proportional hazard regression model. The HRs of each type of drug could be explained as follows: in any given month, if a patient used the given type of drug, the risk of acute pancreatitis would averagely increase $(\mathrm{HR}>1) /$ decrease $(\mathrm{HR}<1)$ compared with a patient who 
Table 1 Baseline characteristics and follow-up status of the primary Sjogren's syndrome (pSS) cohort and the age-matched and sex-matched comparison cohort

\begin{tabular}{|c|c|c|c|}
\hline Group & $\begin{array}{l}\text { Comparison cohort } \\
(n=37872)\end{array}$ & $\begin{array}{l}\text { pSS cohort } \\
(n=9468)\end{array}$ & \\
\hline Variable & n (\%) & n (\%) & p Value* \\
\hline Sex & & & 1 \\
\hline Male & $4836(12.77)$ & $1209(12.77)$ & \\
\hline Female & 33036 (87.23) & $8259(87.23)$ & \\
\hline Age, mean (SD) & $55.61(14.33)$ & $55.64(14.26)$ & 0.863 \\
\hline Age groups, years & & & 0.996 \\
\hline$\leq 50$ & 12945 (34.18) & $3241(34.23)$ & \\
\hline $51-65$ & $14643(38.66)$ & $3658(38.64)$ & \\
\hline$>65$ & $10284(27.15)$ & $2569(27.13)$ & \\
\hline \multicolumn{4}{|l|}{ Baseline comorbidity } \\
\hline Alcoholism & $73(0.19)$ & $36(0.38)$ & $<0.001$ \\
\hline Diabetes mellitus & $4327(11.43)$ & $1095(11.57)$ & 0.702 \\
\hline Gallstone & $423(1.12)$ & $272(2.87)$ & $<0.001$ \\
\hline Hepatitis B & $501(1.32)$ & 321 (3.39) & $<0.001$ \\
\hline Hepatitis C & $324(0.86)$ & $367(3.88)$ & $<0.001$ \\
\hline Hyperlipidaemia & $4716(12.45)$ & $1498(15.82)$ & $<0.001$ \\
\hline Hypertriglyceridaemia & $187(0.49)$ & $59(0.62)$ & 0.117 \\
\hline No of comorbidities $†$ & & & $<0.001$ \\
\hline 0 & 30028 (79.29) & $6674(70.49)$ & \\
\hline 1 & $5512(14.55)$ & $2108(22.26)$ & \\
\hline 2 & $2217(5.85)$ & $616(6.51)$ & \\
\hline$\geq 3$ & $115(0.30)$ & $70(0.74)$ & \\
\hline Follow-up duration, mean (SD) & $4.73(2.78)$ & $4.64(2.78)$ & 0.005 \\
\hline No of acute pancreatitis (AP) & $105(0.28)$ & $44(0.46)$ & 0.005 \\
\hline AP incidence per 100000 person-years & 58.56 & 100.05 & 0.004 \\
\hline
\end{tabular}

did not use the given type of drug. The results of all statistical tests were considered significant if the two-sided $p$ value was $\leq 0.05$.

\section{RESULTS}

Baseline characteristics of the study population

During the study period, a total of 13673 patients with SS were identified. We excluded 3911 patients with secondary SS, 38 patients with incomplete age or sex information, 59 patients aged $<18$ years, 139 patients with a history of acute pancreatitis and 58 with CP before the enrolment date. In total, 9468 patients with pSS were enrolled. We randomly selected and studied age-matched and sex-matched non-pSS controls with the same exclusion criteria, who were four times the number of patients with pSS (table 1). The mean age of patients with pSS was 55.6 years and the majority $(87.23 \%)$ was women. The mean follow-up period of pSS and matched cohorts was 4.64 and 4.73 years, respectively. In the pSS cohort, hyperlipidaemia, gallstones and viral hepatitis (B or $\mathrm{C})$ at baseline were more prevalent $(\mathrm{p}<0.001)$. During the follow-up period, the pSS cohort had a significantly higher incidence of acute pancreatitis $(0.46 \%$ vs $0.28 \%$; $\mathrm{p}=0.005)$ and a higher incidence rate of acute pancreatitis (100 vs 58.6 per 100000 person-years) than the control cohort. Figure 1 shows the Kaplan-Meier analysis, which also revealed a significantly higher cumulative incidence of acute pancreatitis in patients with pSS compared with that in matched controls $(\log$-rank test $\mathrm{p}=0.0025)$.

Comorbidities and acute pancreatitis based on univariate and multivariate Cox proportional hazard analyses

The HR of developing acute pancreatitis during the follow-up period was 1.71 (95\% CI 1.20 to 2.43) in patients with pSS compared with that in patients with 


\section{Cumulative Incidence Plot}

For Acute Pancreatitis

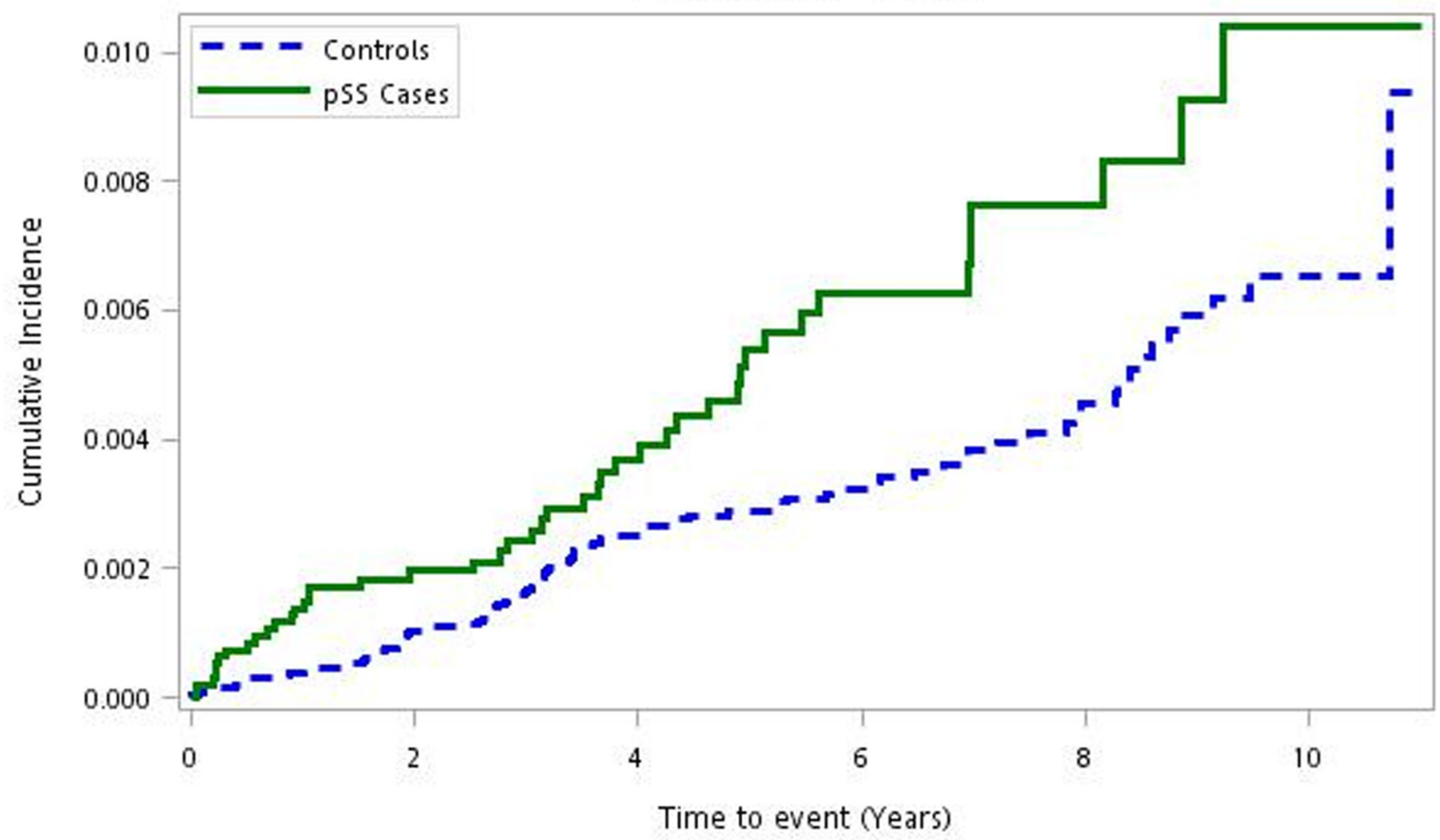

Figure 1 Cumulative incidence of acute pancreatitis in the primary Sjogren's syndrome (pSS) cohort and the comparison cohort.

non-pSS (table 2). After adjustment for patients' sex, age and other comorbidities, the hazard of developing acute pancreatitis during the follow-up period was 1.48 (95\% CI 1.07 to 2.193) times greater in patients with pSS compared with that in patients with non-pSS. This finding suggests that pSS is an independent risk factor for acute pancreatitis. In addition, older age, DM and gallstones increased the risk of acute pancreatitis (aHR 1.61, 2.39 and 5.49, respectively).

\section{Risk factors for acute pancreatitis in patients with pSS}

In patients with $\mathrm{pSS}$, the univariate Cox regression model revealed that male sex, age more than 65 years, DM, gallstone, daily steroids over $5 \mathrm{mg}$ prednisolone equivalent, and time-dependent DMARDs of hydroxychloroquine and cyclophosphamide were significant factors associated with acute pancreatitis (table 3). The multivariate Cox regression model indicated that statistically significant risk factors for acute pancreatitis included age more than 65 years (aHR 2.92, 95\% CI 1.27 to 6.75), gallstones (aHR $5.05,95 \%$ CI 2.10 to 12.16 ), daily steroids over $5 \mathrm{mg}$ prednisolone equivalent (aHR 7.66, 95\% CI 3.71 to 15.84) and cyclophosphamide use (aHR 5.27, 95\% CI 1.16 to 23.86), whereas hydroxychloroquine use reduced the risk (aHR $0.23,95 \%$ CI 0.09 to 0.55 ).

\section{DISCUSSION}

This nationwide, population-based study in Taiwan demonstrated that 9468 patients with pSS had a significantly higher risk of acute pancreatitis compared with the risk in 37872 matched controls, with an aHR of 1.48 after adjustment for age, sex and comorbidities. The incidence rate of acute pancreatitis in patients with pSS was 100.05 per 100000 person-years. The risk factors for acute pancreatitis included age more than 65 years, gallstones, daily steroids over $5 \mathrm{mg}$ prednisolone equivalent, cyclophosphamide use and no hydroxychloroquine use.

To the best of our knowledge, this is the first nationwide population-based cohort study that has demonstrated that patients with SS have an increased risk of acute pancreatitis. The validity of this study is supported by the stringent study design. First, patients with catastrophic illness certification for pSS can be exempted from copayment in the NHI system. The verification requires fulfilment of the AECG criteria after peer review. We also excluded patients diagnosed with other autoimmune diseases. Thus, we believe that our pSS cohort is exhaustive and reliable. Second, this national large cohort was less vulnerable to selection bias and was suitable for studying rare complications and related risk factors. 
Table 2 Cox regression analysis for the risk of acute pancreatitis

\begin{tabular}{|c|c|c|c|c|}
\hline \multirow[b]{2}{*}{ Variable } & \multicolumn{2}{|l|}{ Univariate analysis } & \multicolumn{2}{|c|}{ Multivariate analysis* } \\
\hline & HR $(95 \% \mathrm{Cl})$ & p Value & HR $(95 \% \mathrm{Cl})$ & p Value \\
\hline Primary Sjogren's syndrome & 1.71 (1.20 to 2.43$)$ & 0.003 & 1.48 (1.03 to 2.12$)$ & 0.034 \\
\hline \multicolumn{5}{|c|}{ Age groups, years (reference: age $\leq 50$ ) } \\
\hline $51-65$ & 1.93 (1.20 to 3.12$)$ & 0.007 & 1.61 (0.99 to 2.12$)$ & 0.055 \\
\hline \multicolumn{5}{|l|}{ Baseline comorbidity } \\
\hline Diabetes mellitus & 3.30 (2.30 to 4.73$)$ & $<0.001$ & 2.39 (1.65 to 3.46$)$ & $<0.001$ \\
\hline Gallstone & 7.78 (4.56 to 13.27$)$ & $<0.001$ & 5.49 (3.19 to 9.46$)$ & $<0.001$ \\
\hline Hepatitis B & 2.27 (0.93 to 5.53$)$ & 0.072 & 1.84 (0.74 to 4.54$)$ & 0.189 \\
\hline Hepatitis C & 3.39 (1.59 to 7.25$)$ & 0.002 & 2.06 (0.95 to 4.51$)$ & 0.069 \\
\hline \multicolumn{5}{|l|}{ No of comorbidities } \\
\hline 0 & 1 & & & \\
\hline 1 & 2.51 (1.72 to 3.68$)$ & & & \\
\hline$\geq 2$ & 4.97 (3.24 to 7.65$)$ & & & \\
\hline
\end{tabular}

*All variables, except number of comorbidities, were selected using stepwise Cox regression analysis with entry and retention criteria at $\mathrm{p} \leq 0.2$ in multivariate analysis.

Table 3 Cox regression analysis for the risk of acute pancreatitis among patients with primary Sjogren's syndrome

\begin{tabular}{|c|c|c|c|c|}
\hline \multirow[b]{2}{*}{ Variable } & \multicolumn{2}{|c|}{ Univariate analysis } & \multicolumn{2}{|c|}{ Multivariate analysis* } \\
\hline & HR $(95 \% \mathrm{Cl})$ & p Value & HR $(95 \% \mathrm{Cl})$ & p Value \\
\hline Sex (male) & $2.44(1.26$ to 4.74$)$ & 0.009 & $1.64(0.82$ to 3.26$)$ & 0.161 \\
\hline $51-65$ & 1.42 (0.58 to 3.48$)$ & 0.441 & $1.17(0.47$ to 2.90$)$ & 0.739 \\
\hline$>65$ & 4.22 (1.89 to 9.39$)$ & $<0.001$ & 2.92 (1.27 to 6.75$)$ & 0.012 \\
\hline Gallstone & $5.60(2.37$ to 13.24$)$ & $<0.001$ & 5.05 (2.10 to 12.16$)$ & $<0.001$ \\
\hline Hepatitis B & 2.28 (0.71 to 7.37$)$ & 0.168 & - & - \\
\hline Hepatitis C & $2.21(0.79$ to 6.20$)$ & 0.130 & - & - \\
\hline Hyperlipidaemia & 1.10 (0.49 to 2.47$)$ & 0.817 & - & - \\
\hline \multicolumn{5}{|l|}{ Time-dependent drug effect } \\
\hline Hydroxychloroquine & 0.26 (0.11 to 0.62$)$ & 0.002 & 0.23 (0.09 to 0.55$)$ & 0.001 \\
\hline Cyclophosphamide & 9.61 (2.26 to 39.27$)$ & 0.002 & 5.27 (1.16 to 23.86$)$ & 0.031 \\
\hline Azathioprine & $1.88(0.45$ to 7.78$)$ & 0.384 & - & - \\
\hline Ciclosporin & NA & NA & - & - \\
\hline Sulfasalazine & NA & NA & - & - \\
\hline Methotrexate & NA & NA & - & - \\
\hline Mycophenolate mofetil & NA & NA & - & - \\
\hline
\end{tabular}

${ }^{*}$ Age groups, sex and other variables with $p<0.05$ were selected in multivariate analysis.

$\mathrm{NA}$, not available (did not converge). 
The prevalence rate of acute pancreatitis in our pSS cohort was $0.46 \%$, which is similar to the result obtained in a cohort study in Spain. ${ }^{8}$ The largest case series reported five cases of pSS-related acute pancreatitis among 1010 patients with pSS $(0.5 \%)$. Furthermore, our study revealed that the pSS cohort had a significantly higher risk of acute pancreatitis than age-matched and sex-matched controls. Comorbidities such as alcoholism, DM, hepatitis B, hepatitis C and hyperlipidaemia had significantly higher prevalence rates in our pSS cohort. However, we believe the higher rates of these comorbidities might due to the characteristics associated with patients with pSS or, more likely, a higher diagnosis rate caused by the higher medical usage rate in the pSS cohort. Moreover, our conservative analysis revealed pSS to be a significant independent risk factor for acute pancreatitis after correcting for these comorbidities.

The risk factors for older age and gallstone were common between our pSS cohort and the general population. In addition, we found that medication use was associated with acute pancreatitis in patients with pSS. Limited studies have examined the association between steroids or DMARDs and acute pancreatitis. Immunosuppressants such as azathioprine and ciclosporin have been implicated as causes of pancreatitis in several case reports. Badalov et al found that cyclophosphamide use was associated with acute pancreatitis, which was also observed in our pSS cohort. ${ }^{13}$ In this claims data-based study, it was unclear whether cyclophosphamide increased the risk through drug toxicity or was a marker of systemic manifestations in the pSS cohort. However, autoimmune-related inflammation was suspected on the basis of the association with higher daily steroid use and no HCQ use. A similar finding was obtained for SLE-related acute pancreatitis and AIP. ${ }^{1415}$ AIP was found to be associated with autoimmune diseases (SS, rheumatoid arthritis, primary sclerosing cholangitis and inflammatory bowel disease) ${ }^{16}$ Vascular damage, including vasculitis, intimal thickening, immune complex deposition and occlusion of arteries and arterioles); autoantibody production and abnormal cellular immune response may be responsible for the development of pancreatitis. ${ }^{17}$ Patients with a higher daily steroid dose and cyclophosphamide therapy and without HCQ use might have a higher risk of autoimmune-related pancreatitis.

Hepatitis $\mathrm{C}$ virus (HCV) is associated with both SS and acute pancreatitis and might be an important confounder in our study. Furthermore, patients with HCV should be excluded according to the 2002 AECG criteria. However, the pathogenesis of the association between SS and HCV is not fully known and whether the sicca syndrome in patients with HCV is due to pSS, secondary SS or only SS-like symptoms remains controversial. ${ }^{18}$ Moreover, neither correcting $\mathrm{HCV}$ in the multivariate Cox model nor the analysis after excluding patients with pSS with prior HCV and their matched controls resulted in different outcome. Thus, the initial study design was not altered and HCV was not excluded.
Our study has clinical implications. First, acute pancreatitis is a rare complication among patients with pSS and should be considered one of the differential diagnoses of abdominal pain. Second, without using hydroxychloroquine might be considered as a risk factor, particularly among those with higher daily steroid use or cyclophosphamide treatment. Administrative databases enable population-based epidemiological studies; however, limitations exist. First, some data are unavailable in this claims-based dataset. Data on alcohol intake and smoking, the major risk factors of acute pancreatitis, were unavailable in our study. We still included 'alcoholism' in our study, which was defined as the presence of related ICD-9CM codes prior to the diagnosis of pSS. Unlike other comorbidities, alcoholism was easily underestimated because we could only identify those went to a doctor. Thus, we believed that the coding of alcoholism was influenced by the medical use, which resulted in a significant lower rate of alcoholism in the control cohort. Moreover, no reports have mentioned the relationship between pSS and alcoholism. Dryness of the oral mucosa in pSS can result in alcohol intolerance. However, we do not know whether average alcohol consumption decreased after the onset of dry mouth. Furthermore, because of the lack of data on the severity of pSS, laboratory results and indications for medication use, we could not determine the mechanism of pancreatitis. Second, IgG4-related disease may involve salivary and lacrimal glands and AIP. ${ }^{19}$ However, in the National Health Insurance Research Database (NHIRD), the certification of pSS requires a positive anti-Ro or/ and anti-La antibodies or a positive lip biopsy. In addition, the observed lower risk in those using HCQ, which is not beneficial to IgG4-related disease, also implied the limited effect of IgG4-related disease in our study.

In conclusion, we demonstrated that patients with pSS had a higher risk of acute pancreatitis, and the magnitude of hazard in the pSS-affected population was $48 \%$ higher than that in the non-pSS population. On the basis of these findings, acute pancreatitis should be considered one of the differential diagnoses when related symptoms present.

\section{Author affiliations}

${ }^{1}$ Division of Rheumatology, Immunology and Allergy, Department of Internal Medicine, Taipei Medical University Hospital, Taipei, Taiwan

${ }^{2}$ Division of Allergy, Immunology and Rheumatology, Department of Internal Medicine, School of Medicine, College of Medicine, Taipei Medical University, Taipei, Taiwan

${ }^{3}$ Division of Allergy, Immunology and Rheumatology, Department of Internal Medicine, Shuang Ho Hospital, Taipei Medical University, New Taipei City, Taiwan ${ }^{4}$ Department of Primary Care Medicine, Taipei Medical University Hospital, Taipei, Taiwan

${ }^{5}$ Department of General Medicine, School of Medicine, College of Medicine, Taipei Medical University, Taipei, Taiwan

${ }^{6}$ Biostatistics Centre College of Management, Taipei Medical University, Taipei, Taiwan

${ }^{7}$ Graduate Institute of Data Science, College of Management, Taipei Medical University, Taipei, Taiwan

Contributors C-CC contributed to the conception and design of the work, drafting of the article, revision of the article critically for crucial intellectual content, and final 
approval of the version to be published. Y-SC contributed to interpretation of data, revision of the article critically for crucial intellectual content, and final approval of the version to be published. S-HW and S-YL contributed to the analysis of data, revision of the article critically for crucial intellectual content, and final approval of the version to be published. Y-HC contributed to the analysis of the data, drafting of the article, and final approval of the version to be published. J-HC designed the study and conceived the work, completed the analysis, revised the article critically for crucial intellectual content and corresponded for final approval of the version to be published. All authors have disclosed any potential competing financial interests regarding the submitted article.

Competing interests None declared.

Patient consent Obtained.

Provenance and peer review Not commissioned; externally peer reviewed.

Data sharing statement Extra data can be accessed via the Dryad data repository at http://datadryad.org/ with the doi:10.5061/dryad.22h7c.

Open Access This is an Open Access article distributed in accordance with the Creative Commons Attribution Non Commercial (CC BY-NC 4.0) license, which permits others to distribute, remix, adapt, build upon this work non-commercially, and license their derivative works on different terms, provided the original work is properly cited and the use is non-commercial. See: http://creativecommons.org/ licenses/by-nc/4.0/

(c) Article author(s) (or their employer(s) unless otherwise stated in the text of the article) 2017. All rights reserved. No commercial use is permitted unless otherwise expressly granted.

\section{REFERENCES}

1. Mavragani CP, Moutsopoulos NM, Moutsopoulos HM. The management of Sjögren's syndrome. Nat Clin Pract Rheumatol 2006;2:252-61.

2. Ramos-Casals M, Font J. Primary sjogren's syndrome: current and emergent aetiopathogenic concepts. Rheumatology 2005;44:1354-67.

3. Hernández-Molina G, Michel-Peregrina ML. [Sjögren's syndrome and pancreatic affection]. Reumatol Clin 2011;7:130-4.
4. Yadav D, Lowenfels AB. The epidemiology of pancreatitis and pancreatic Cancer. Gastroenterology 2013;144:1252-61.

5. Fukui T, Okazaki K, Yoshizawa $\mathrm{H}$, et al. A case of autoimmune pancreatitis associated with sclerosing cholangitis, retroperitoneal fibrosis and sjögren's syndrome. Pancreatology 2005;5:86-91.

6. Ohara H, Nakazawa T, Sano H, et al. Systemic extrapancreatic lesions associated with autoimmune pancreatitis. Pancreas 2005;31:232-7.

7. Pickartz T, Pickartz H, Lochs H, et al. Overlap syndrome of autoimmune pancreatitis and cholangitis associated with secondary Sjögren's syndrome. Eur J Gastroenterol Hepatol 2004;16:1295-9.

8. Ramos-Casals M, Solans R, Rosas J, et al. Primary sjögren syndrome in Spain: clinical and immunologic expression in 1010 patients. Medicine 2008;87:210-9.

9. Matsumoto J, Harigai M, Nishimagi E, et al. [A case of Sjögren's syndrome and systemic sclerosis complicated with acute pancreatitis]. Ryumachi 2000;40:620-6.

10. Weng MY, Huang YT, Liu MF, et al. Incidence of Cancer in a nationwide population cohort of 7852 patients with primary Sjogren's syndrome in Taiwan. Ann Rheum Dis 2012;71:524-7.

11. Shen HN, Lu CL, Li CY. Epidemiology of first-attack acute pancreatitis in Taiwan from 2000 through 2009: a nationwide population-based study. Pancreas 2012;41:696-702.

12. Shen HN, Lu CL, Li CY. Effect of diabetes on severity and hospital mortality in patients with acute pancreatitis: a national populationbased study. Diabetes Care 2012;35:1061-6.

13. Badalov N, Baradarian R, Iswara K, et al. Drug-induced acute pancreatitis: an evidence-based review. Clin Gastroenterol Hepatol 2007:5:648-61.

14. Saab S, Corr MP, Weisman MH. Corticosteroids and systemic lupus erythematosus pancreatitis: a case series. $J$ Rheumatol 1998;25:801-6.

15. Kapoor D, Mendez E, Espinoza LR. Corticosteroids and SLE pancreatitis. J Rheumatol 1999;26:1011-2.

16. Vonlaufen A, Wilson JS, Apte MV. Molecular mechanisms of pancreatitis: current opinion. $J$ Gastroenterol Hepatol 2008:23:1339-48.

17. Lariño Noia J, Macías García F, Seijo Ríos S, et al. Pancreatitis and systemic lupus erythematosus. Rev Esp Enferm Dig 2009;101:571-9.

18. Yeh CC, Wang WC, Wu CS, et al. Association of Sjögrens syndrome in patients with Chronic Hepatitis virus infection: a Population-Based analysis. PLoS One 2016:11:e0161958.

19. Palazzo E, Palazzo C, Palazzo M. IgG4-related disease. Joint Bone Spine 2014;81:27-31. 